\title{
ON THE PHENOMENON OF DIGITAL INEQUALITY
}

\author{
Mysore Ramaswamy, Southern University, Baton Rouge, LA, mysore@acm.org
}

\begin{abstract}
The last few decades have seen tremendous growth in the field of information and communication technology (ICT) that have affected all aspects of modern life. Contemporary society has been variously called 'Information Society,' 'Digital Economy,' and 'Knowledge Economy.' This is reflected in the degree to which we are dependent on electronic or digital means even to execute our daily routine activities. Unfortunately, this convenience has not reached all segments of our society. Globally speaking, only one billion out of seven billion has access to the Internet. Here in the United States, 27 percent of the population lacks access to the Internet, according to a study completed last year by the Pew Internet and American Life Project. In spite of research on digital divide and digital inequality, there is still a need for a comprehensive analysis that combines ICT Innovations, ICT Access, and different skill levels in ICT Use. In this paper, we study the phenomenon of digital inequality through these three lenses and analyze the factors that aid in building a more inclusive e-society where there is no room for digital inequality.
\end{abstract}

Keywords: Digital Divide, Digital Inequality, E-Society, Information and Communication Technology (ICT).

\section{INTRODUCTION}

In spite of tremendous innovations in the field of information and communication technology (ICT), these benefits have not reached all segments of our society. All governments across the world desire their citizens and businesses to communicate through electronic means for the obvious reasons of efficiency and effectiveness. The number of Internet users has increased exponentially during the last two decades. In spite of this phenomenon, large gaps exist between groups regarding their ability to use information and communication technologies effectively [6, 9, 13]. Digital exclusion may result from a lack of digital literacy, from economic or technical barriers to Internet access, or from a lack of capabilities to use efficiently the new services and facilities linked to information and communication technologies [12]. This digital exclusion results in not benefiting from knowledge resources, the potential of new information and communication services, new job opportunities and better access to employment, and overcoming barriers of distance and mobility.

Much has been written about the potential of information technology to 'revolutionize' society, particularly in the context of their role as catalysts of the 'Information Revolution'. This 'revolution' is often juxtaposed with its predecessor, the Industrial Revolution, usually for the purpose accentuating the idea that communication networks are as integral to the process of development as was the birth and development of industry in the $19^{\text {th }}$ century. While it is the question of access that has risen to the forefront of development agendas in the context of the digital exclusion, much work remains to be done in analyzing and understanding how these technologies are utilized and applied to bring about expected revolutionary societal and economic changes and improvements.

In the early stages, the Internet use relied on dial-up connections that offer limited capacity (56Kbps or less) and intermittent connectivity. The first generation of advanced services with continuous connectivity and expanded capacity (200Kbps to 1Mbps) began to be deployed in the latter half of the 1990s. These include DSL services offered over telephone company copper wires, cable modem services offered over cable television facilities, and a small but growing number of wireless services (satellite and terrestrial). However, there are still significant portions of population who do not have access to these services.

The first challenge to a more inclusive e-society - where there is no digital inequality - is the need for a robust telecommunication infrastructure that can support all digital communication. Globally speaking, only one billion out 


\section{Issues in Information Systems}

Volume 13, Issue 1, pp. 240-246, 2012

of seven billion has access to the Internet. Here in the United States, 27 percent of the population lacks access to the Internet, according to a study completed last year by the Pew Internet and American Life Project. Among those who do have access, about 30 percent still rely on slow dial-up connections. The second and perhaps harder challenge is to mitigate the problem of digital exclusion. The rest of the paper is organized as follows. The next section discusses the first order digital divide where the focus is on the issue of digital access. Issues of ICT skills or what is commonly known as second order digital divide are discussed in the subsequent section. The next section looks at ways to bridge the digital gap. The conclusions and future research directions are presented in the last section.

\section{FIRST ORDR DIGITAL DIVIDE}

In this section, we study the following two critical components of digital inequality, viz. digital infrastructure, and interaction content. Digital infrastructure refers to the mechanism through which users access the cyberspace. Interaction content is what drives the users to go online.

In the early stages, the Internet use relied on dial-up connections that offer limited capacity (56Kbps or less) and intermittent connectivity. The first generation of advanced services with continuous connectivity and expanded capacity (200Kbps to 1Mbps) began to be deployed in the latter half of the 1990s. These include DSL services offered over telephone company copper wires, cable modem services offered over cable television facilities, and a small but growing number of wireless services (satellite and terrestrial). However, there are still significant portions of population who do not have access to these services.

Broadband Internet connectivity is viewed as an important part of the infrastructure required to ensure effective ecommerce. The Organization for Economic Cooperation and Development (OECD) states that as of December 2005, the number of broadband subscribers in the United States was 16.8\% (total subscribers: 49,391,060). The breakup is as follows: DSL: 6.5\%, Cable: $9.0 \%$, Other: $1.3 \%$. United States ranks $12^{\text {th }}$ among the industrialized countries in the list of broadband subscribers per 100 inhabitants. By ensuring high-speed access and 'always-on' connections, broadband technologies substantially change the patterns of Internet usage [7]. Moreover, whether many of these services should even be classified as 'broadband' has been questioned. The Federal Communications Commission (FCC) defines 'advanced services' as capable of providing more than $200 \mathrm{Kbps}$ on both directions. By that definition, many cable and DSL systems do not qualify, as they are constrained to upstream bandwidths of $128 \mathrm{Kbps}$ or less. At a mere four times the bit rate, $200 \mathrm{Kbps}$ represents only an incremental step from a dial-up modem. Much higher capacity access networks offering services in the 10s to 100s of Mbps (or more) per household will be needed to fully unleash the full potential of advanced communications infrastructure.

Local governments have the following two important roles to play in the development of broadband infrastructure: (i) financier and (ii) infrastructure developer [3]. Financial incentives can be aimed at stimulating supply, demand, or both. Subsidies to commercial providers can be in the form of outright grants, low-cost loans, or tax incentives. Subsidies to users are typically temporary in nature, or targeted at disadvantaged groups. For example, LaGrange, Georgia gave away WebTV equipment and service for a one year period in an attempt to get more of their socioeconomically citizens online [7]. Unfortunately, the attempt was largely unsuccessful, reflecting barriers to adoption that go beyond the cost of access, as well as unpopular limitations of the chosen technology - the WebTV devices used had no print capability. Broadband related subsidies appear to be more common at state and federal levels, where budgets are significantly large. For example, Pennsylvania administers a \$3.3million digital divide grant program based on federal funds from the Temporary Assistance to Needy Families (TANF) program. One of their grants was $\$ 457,000$ to the Glendale School District (located in economically disadvantaged coal country) to extend wireless broadband Internet access from one school to neighboring schools as well as citizens, and to give laptops to community members who partake of training opportunities [5].

The other strategy that local governments can pursue to stimulate broadband is to develop one or more aspects of the necessary infrastructure themselves. Based on an assessment of the communications needs and existing resources available to different groups of users, government can prioritize infrastructure developments for itself, local 


\section{Issues in Information Systems}

Volume 13, Issue 1, pp. 240-246, 2012

businesses, and citizens. A local government can deploy a network for its own needs, and later exploit any excess capacity to offer services to nearby businesses.

Wireless networks add their own complexity to the mix. Last year, Google while testing its first municipal Wi-Fi network in its hometown, Mountain View, Calif., using transmitters attached to street lamps, observed that people using Google's network could get online at home only by holding their laptops against a window. This was to be expected as using municipal Wi-Fi for residential coverage was "the equivalent of expecting street lamps to light everyone's homes."

The second critical component of e-society refers to the digital content that users can access User interactions with digital or electronic means have been grouped in a number of ways [15]. In the present analysis, it will be useful to categorize them in the following way: information services, communication services, and transaction services. A more inclusive e-society has to provide more useful digital interactions to a larger segment of the society, especially in the third category of transaction services.

In the context of e-inclusion, under the category of transaction services, government-to-citizen interactions of eGovernment become very significant. In order to build a more inclusive e-society, we need to address these questions:

- How can we make e-Government truly citizen-centric?

- Do we have objectives that are correct and realistic?

- How well are we achieving these objectives fully and cost effectively?

- Are these objectives jointly determined and agreed upon by the citizens and the government agencies?

We need better models of the processes used to plan, fund, develop, implement, operate, and evaluate successful eGovernment in various political and social, cultural, and economic contexts [3].

Some of the above concerns are addressed by the e-Governance cube [14]. They propose the formation of an egovernance cube with the following three axes: entity type on the $\mathrm{x}$-axis, processing complexity on the $\mathrm{y}$-axis, and perceived value on the z-axis. The e-governance cube provides a means of evaluating an extensive (if not comprehensive) series of government-constituent interactions. Depending upon which of the 27 sub-cubes contains the given transaction of interest, we are now able to make inferences on the potential of that transaction for its ease of conversion to e-governance. This naturally opens up a wide arena of analysis, particularly for others who wish to specialize and focus specifically on the dynamics and characteristics of specific e-governance transactions. In the context of the e-governance cube model, the further one moves away from the origin, the more resistance one encounters. From the e-inclusion view point, the processes that are valued high on the z-axis (perceived value) need to be addresses first.

\section{SECOND ORDER DIGITAL DIVIDE}

Second order digital divide refers to the different ways people use ICT based on their skill levels [16]. One of the most important aspects of digital inequality has to do with differences in computer skill levels [4]. The way in which people connect to the Internet also contributes to the second-order effect. Users with broadband connections are more likely to spend more time on the Internet than those with dial-up connections. In addition, the ability to connect via a broadband connection impacts what users do online. Pew Internet Data suggests that more experienced users are much more likely to do online transactions and manage their money online compared to more recent adopters of the Internet. Pew Internet Data also indicates that more experienced users are likely to have a higher socio-economic status. People with superior technical access and usage skills are emerging as a class of online elite users who can be grouped as "Netizens." These people are more likely to engage in online commerce activities and use powerful e-commerce functionalities such as recommender services and online auctions.

Figure 1 indicates a typology of users as suggested by Brotcorne [1\}. This typology of users is based on two critical dimensions: initial motivation and perceived usefulness. Motivation or the initial impetus refers to the contextual 


\section{Issues in Information Systems}

Volume 13, Issue 1, pp. 240-246, 2012

factors that inspire an individual to become interested in computers and/or the Internet and to become a user. The motivation or initial impetus was characterized by three levels of constraint: high, medium, and low. The perceived usefulness can be deduced from the usage framework and the usage territory, i.e., the types and the nature of the ICT uses. Usefulness is qualified as "perceived" because it is not determined in a normative manner, but is expressed by the user. Three levels of perceived usefulness have been differentiated: high, medium, and low.

User trajectories are structured by the above mentioned two dimensions - the motivation or initial impetus and the perceived usefulness - in terms of attitude towards learning, a quest for autonomy and the ability to assimilate the behavior pattern expected from a user who is part of the information society. The combination of these two dimensions is used by Brotcorne [1] to identify eight ideal types, which are differentiated by the initial impetus and the usage territory. Type 1 user is classified as 'conscientious apprentice' who likes to learn but requires support and guidance. Users in this type have a conscious desire for professional integration and a need for social recognition. They have a positive and utilitarian relation with ICT. This type of profile is not particularly different in terms of age, educational or activity level variables. It may perhaps be more feminine, professionally active and middle-aged. Type 2 user is classified as 'distant skillful user.' This user has had to familiarize herself with ICT for professional reasons. The usage territory is extended at work and limited at home. The professional life has little impact on her private life. These users do not feel the need to use ICT at home and do not see why they should. This is a typically feminine profile, professionally active, older, from all levels of education.

Type 3 user can be classified as "clairvoyant follower." Typically, such a user has adopted ICT under gentle pressure from his or her entourage, but this quickly turned into a personal interest. The Internet provides support for the existing activities for the users of this group. They attribute positive symbolic value to ICT but in a controlled space and with a reflective attitude. A concern for autonomy and a proactive attitude also characterize this type of profile, which is essentially masculine, and on average these users are older. Type 4 users can be called 'assiduous users by default.' The users in this category have accepted ICT during their school years but the professional need constituted the decisive impetus. These users use the Internet in all its forms and communicate a lot to break free from their isolation. They attribute considerable symbolic value to ICT: it is their link with the world. This profile is usually young, socially vulnerable, often in situations of transition.

\begin{tabular}{|c|c|c|c|c|}
\hline \multicolumn{2}{|c|}{} & \multicolumn{3}{|c|}{ Perceived Usefulness } \\
\cline { 3 - 5 } & $\begin{array}{c}\text { Strong } \\
\text { Constraint }\end{array}$ & High & Medium & Low \\
\hline \multirow{3}{*}{$\begin{array}{c}\text { Motivation / } \\
\text { Social } \\
\text { Impetus }\end{array}$} & & $\begin{array}{c}\text { Type 1 } \\
\text { The Conscientious } \\
\text { Apprentice }\end{array}$ & $\begin{array}{c}\text { Type 2 } \\
\text { The Distant Skillful } \\
\text { User }\end{array}$ \\
\cline { 2 - 5 } & Medium & & Type 3 & Type 4 \\
& Constraint & $\begin{array}{c}\text { The Clairvoyant } \\
\text { Follower }\end{array}$ & $\begin{array}{c}\text { The Assiduous User by } \\
\text { Default }\end{array}$ & $\begin{array}{c}\text { Type 5 } \\
\text { The Resigned } \\
\text { Skeptic }\end{array}$ \\
\cline { 2 - 5 } & Low to no & Type 6 & Type 7 & Type 8 \\
& Constraint & The Expert Routine & The Seduced Curious & The Conformist \\
& & User & & Consumer \\
\hline
\end{tabular}

Figure 1. A Typology of Users (Adapted from [1])

Type 5 consists of users who can be classified as 'resigned skeptics.' They would have started using ICT without great enthusiasm, under moderate pressure from professional colleagues. They use ICT sparingly. Their learning process is often laborious and dispassionate. They are resistance fighters who have given up but aware of their withdrawn position. Sometimes, these users attribute negative symbolic value to ICT. The vast majority in this category have a degree in higher education. Type 6 users can be classified 'expert routine users.' With early familiarization and sustained curiosity, these assiduous users have incorporated ICT usage in their daily lives. They 


\section{Issues in Information Systems}

Volume 13, Issue 1, pp. 240-246, 2012

attribute a positive symbolic value to ICT in combination with a proactive attitude. This profile is typically young, at work, both feminine and masculine.

Type 7 user profile refers to the 'secluded curious.' These users are moved by a desire to discover ICT, which progressively takes on a meaning in their daily lives. They tinker around with ICT, which take on a highly positive symbolic meaning to them. Typically these users are independent. This profile is rather masculine and active. Type 8 consists of 'conformist consumers.' They express a need for social affiliation through the ownership of symbolic high-tech objects. Their use of the Internet is intensive and concentrated in the areas of entertainment and communication. ICT is not part of their professional environment. They do not feel the need for training. In this category, the majority are aged 30 years or less.

The above classification of users helps in understanding how user's attitude for learning is conditioned by perceived usefulness and the value attributed to ICT usage. This type of information will help in developing public policies that are designed to support activities such as training of ICT use, awareness campaigns, public access, certain types of financial or tax aid, a legal frame for online services, etc. Some forms of institutional support are designed for everybody while others are aimed at certain target audiences. The second order digital divide is caused essentially due to varying degrees of ICT skills exhibited by users. Confirmed users acquire and develop their ICT skills through different channels and based on different behaviors. They learn about ICT at school, job training, or through informal types of training such as self-study. A positive and proactive attitude to learning is exhibited by the following types: clairvoyant follower, expert routine user, seduced curious and assiduous user. A positive but reactive attitude to learning is exhibited by the following groups of users: conscientious apprentice, and distant skillful user. The conformist consumers show a distant attitude to learning marked by disinterest. The resigned skeptics have a superficial attitude to learning.

\section{CLOSING THE GAP}

The objective of 'E-inclusion' is to mitigate the effects of digital inequality. According to advocates, e-inclusion has the power to: close the gap between developed and less developed countries; promote democracy and mutual understanding; and empower disadvantaged individuals, such as the poor, the disabled, and the unemployed.

An increasing number of computer equipment and software companies have e-inclusion programs. According to Hewlett-Packard, the purpose of their e-inclusion initiative is "... to close the gap between the technologyempowered communities and the technology-excluded communities on our planet by making it profitable to do so." The company's program is targeting low-income areas in the developing world to improve opportunities for education, healthcare, and employment and deliver access to world markets and consumer credit.

Recent studies of the digital divide confirm that the user education and skills are discriminating factors in the use of e-services. They also indicate that the 'access divide' is narrowing [12]. The effective use of e-services relies on relies on cumulative skills developed through the use itself (cognitive and social resources). The 'second order' digital divide refers to the ability to deal with continuous evolution and changing complexity of e-services.

To attract technology shy or resistant people, awareness campaigns should be expanded in communities at risk of digital exclusion, communicating the specific benefits for disadvantaged people, and using channels that are appropriate for their way of life. Awareness measures should be designed as a first step in an integrated e-inclusion strategy, followed-up by incentives for access and training. Information and communication technology (ICT) producers, information providers and government authorities should be sensitized to the special needs of disadvantaged people in terms of ICT equipment and online content and in terms of opportunities for removing barriers for work.

Some of the other broad range issues emerging in the context of e-inclusion are: 


\section{Issues in Information Systems}

Volume 13, Issue 1, pp. 240-246, 2012

- demand-related issues such as lacking motivation among particular population groups to utilize online services for one's own improvement;

- supply-oriented issues such as lack of recognition of specific requirements certain population groups may have with the services offered;

- regulation-related issues such as restricted availability of the required technical infrastructure (access networks, terminals) among particular population groups.

\section{CONCLUSION}

Innovations in information and communication technologies have rendered electronic means of interactions so ubiquitous that we are indeed living in an e-society. However, some sections of our population do not seem to be fully exploiting the available technology. Broadband availability and appropriate digital content are some of the factors that have become obstacles in reducing digital inequality.

To tap the e-society's potential for disadvantaged people, the following recommendations are offered:

- develop quality checks of responses of public web sites to special needs of disadvantaged people;

- stimulate online activities in the voluntary and non-profit sector;

- include ICT literacy of low-income and low-educated workers in the employment guidelines;

- match online recruitment services with the needs of workers with special needs;

- explore the opportunities of digital TV and mobile communications for e-inclusion purposes.

The policies implemented so far regarding digital inclusion have often been of a missionary nature. They were designed to convince citizens of the individual and collective benefits of the information society. The overarching motivation behind these policies was the democratization of ICT access. In this paper we have analyzed factors such as user trajectories and usage territories. The newly emerging concept of secondary digital divide indicates that the appropriation of ICT does not automatically to social integration. In this context social media have a vital role to play and this will be studied as part of future research.

\section{REFERENCES}

1. Brotcorne, P. et al. (2010). The Second Order Digital Divide. Fondation Travail-Universite asbl, Belgium.

2. Carbo, T. and Williams, J. (2004). Models and Metrics for Evaluating Local Electronic Government Systems and Services. The Electronic Journal of Electronic Government, Vol. 2, No. 1, pp. 99-106.

3. Clark, K. and Baker, P. (2003). Municipal Advanced Telecommunication Infrastructure Project (MuniTIP). Georgia Center for Advanced Telecommunications Technology, OTP Policy Study No. 50103.http://www.gcatt.org/otp/papers/ MuniTIP.pdf

4. Dewan, S. and Riggins, F. J. (2006). The Digital Divide: Current and Future Directions, Journal of the Association for Information Systems, Vol. 6, No. 12, pp. 298-337.

5. Government Technology. (2002). Pennsylvania Targets Digital Divide. http://www.govtech,net/news.news.phtml?docid=2002.1209.33355

6. Hopkins, L. (2005). Making a community network sustainable: The future of the wired high rise, Information Society, 21 (5), 379.

7. Keil, M. et al. (2003). Bridging the Digital Divide: The Story of the Free Internet Initiative in LaGrange, Georgia. Proceedings of the $36^{\text {th }}$ Hawaii International Conference on Information Systems, (10 pages).

8. Keniston, K. (2003). The Four Digital Divides, Sage Publishers, Delhi, India.

9. Koss, F. A. (2001). The Challenges of redressing the digital divide, Journal of International Affairs, 55 (1), 75-90.

10. Kvasny, L. (2002). A Conceptual Framework for Examining Digital Inequality, AMCIs 2002 Proceedings, Paper 246. 


\section{Issues in Information Systems}

Volume 13, Issue 1, pp. 240-246, 2012

11. Lee, H. et al (2003). The growth of broadband and electronic commerce in South Korea: Contributing factors, Information Society, 19 (5), 81-93.

12. Mason, S. M. et al (2003). Applying communication theory to digital divide research, IT and Society, 1 (5).

13. Mistry, J. J. (2005). A conceptual framework for the role of government in bridging the digital divide, Journal of Global Information Technology Management, 8 (3), 28-46.

14. Ramaswamy, M. and Selian, A. (2007). e-Government in Transition Countries: Prospects and Challenges, Proceedings of the $40^{\text {th }}$ Hawaii International Conference on Information Systems, (10 pages).

15. Turban, E. et al, Electronic Commerce: A Managerial Perspective, Prentice Hall, Upper Saddle River, NJ, 2004.

16. Warchauer, M. et al. (2004). Technology and Equity in Schooling: Deconstructing the Digital Divide, Educational Policy, 18(4): 562-588 